\title{
Portable and Projectable Mini PC
}

\author{
VijayaShangamesh $\mathrm{M} \mathrm{A}^{1}$, Anusuya $\mathrm{K} \mathrm{V}^{2}$, Adithya $\mathrm{V}^{3}$ \\ \{vijayashangamesh01@gmail.com ${ }^{1}$, kva.ece@psgtech.ac.in ${ }^{2}$, adiashi816@gmail.com ${ }^{3}$ \} \\ Associate Professor ${ }^{2}$, Department of ECE, PSG College of Technology, Coimbatore. ${ }^{1,3}$
}

\begin{abstract}
The portable computers called laptops are heavy and not compact. The laptops with touch facilities are relatively costlier and they require the user to be in close contact with the screen, causing eye complications. Also, it requires a connection to the projector separately while doing presentations. To address most of these issues, a portable and projectable mini-PC design is proposed. It includes the built-in design of a mini portable screen cum projector. In addition, a user-friendly environment is created using voicecontrolled models to avoid the need for the keyboard and efficient presentation control through hand movements. Moreover, a projector module is built using the principles of the lens. In the hardware, the main module of Raspberry Pi is interfaced with other peripherals. The projector module is built with lenses by applying the principles of the lens. The voice module activated by the CPU remains in an active state. The hand gesture module that uses ESP32 is interfaced with raspberry-pi via Bluetooth.
\end{abstract}

Keywords: Hand movements, Mini-PC, Portable, Projectable, Voice recognition.

\section{Introduction}

Computers, a human invention is the driving source for most of the sectors such as industries, education, and security, etc.., in the world. The laptops being personal computers are easily portable but are not at an affordable cost to the common people. Moreover, the lowquality LCD screens of laptops can lead to eye defects. Hence, a miniaturized PC design is proposed with a projectable screen. Moreover, the design objectives include the control of mini-PC through the human voice and gestures. This design is most suitable for small-scale meetings and conversations, where presentations are done without the need for a projector. The proposed design of the mini-PC is comparatively smaller in size and costlier than the laptops, and the mini projector is smaller in size and cost-efficient than the other usual projectors. Hence, the portable computer is handy with maximum possibilities of utilization with less power and projection in near hand. This makes the importance of the processorbased microcontroller feasible of converting into a mini personal computer with ever expandable memory along 
with voice and gesture modules that replace the traditional keyboard and mouse.

\section{Proposed Design}

In the proposed design of a portable and projectable mini- PC, a common power port is connected to the projector module and the CPU. When the PC is powered on, the projector and the Raspberry Pi (CPU) get the power. The CPU gets booted and the screen gets projected onto the wall with the use of lenses. The Bluetooth microphone should be connected with the Raspberry Pi before using the voice recognition module. After connecting the microphone, the word named 'Flora' wakes up the operation. With this 'Bumble bee', the voice recognition software opens. Commands need to be given to the bumblebee assistant, to enable the execution. The commands to control the keyboard and the mouse are given to the bumblebee voice recognition module. The voice recognition is made off, by using the close command.

The design includes a gesture-based presenter module also. It has a separate power source and at the beginning, the presenter device must be turned $\mathrm{ON}$ and must be connected to the CPU via Bluetooth. The operation corresponding to the gesture is performed and also stored in EEPROM for further use. The proposed mini-PC can be connected to other projectors and screens via the micro- HDMI port given in the Raspberry $\mathrm{Pi} 4 \mathrm{~b}$. The operation is depicted in the flow diagram shown in Fig. 1.

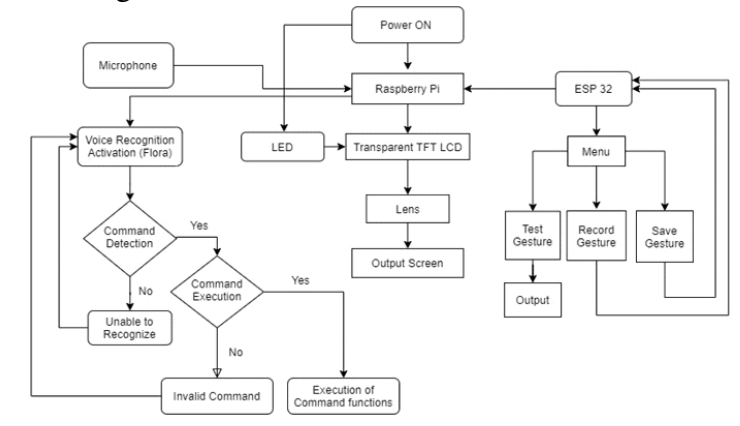

Fig. 1. The working mechanism of the proposed design

\section{Experimental Setup}

The mini-PC design contains three main modules, namely the projector module, voice recognition module, and gesture-based PowerPoint presenter module. All three modules are connected to the CPU (Raspberry Pi $4 \mathrm{~b}$ ) either wired or through wireless mode such as Bluetooth. The projector module consists of a high-power LED, Fresnel lens, convex lens, and TFT LCD. The TFT LCD is a Raspberry Pi LCD shield. When the Raspberry Pi is turned ON, the LCD shield connected to it gets turned ON directly. The TFT LCD shield is made transparent by removing the polarizing layers in the LCD [1]. The basic design of the projector module is shown in Fig.2. 


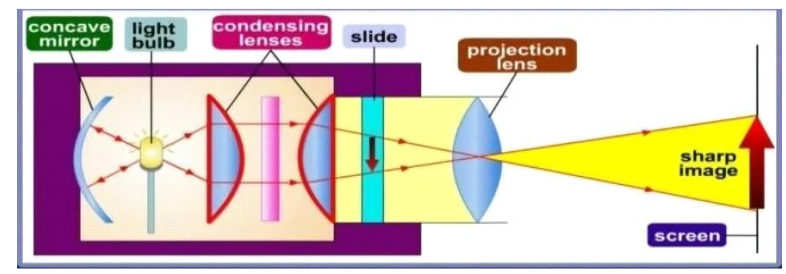

Fig.2. Design of LCD projector

The voice recognition module is the software developed using python libraries. The Google API library helps in both speech-to-text and text-to-speech conversions [2]. The commands to be executed are programmed using python scripts. This Google API uses the cloud to collect data for TTS and STT conversions. The gesture-based presenter module contains ESP

32 microcontroller as the controller, MPU 6050 for gesture detection, and an OLED display for displaying the contents [3]. The MPU 6050 and OLED display is connected to ESP 32 through the I2C protocol. Figure 3 depicts the modular design of Portable and projectable Mini - PC.

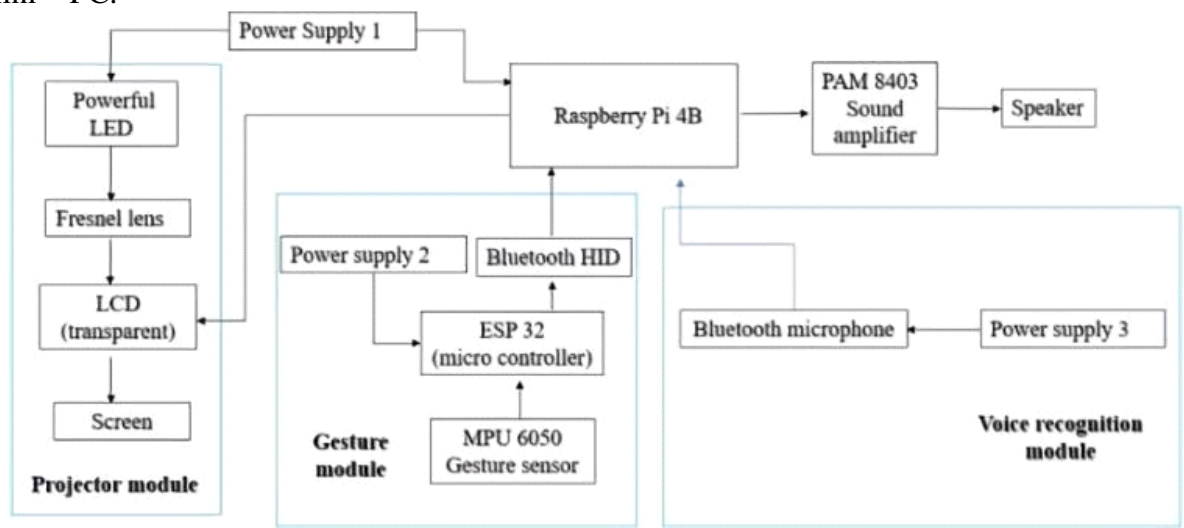

Fig 3. The modular design of Portable and projectable mini-PC.

\section{- Projector module}

A 50W high-power LED is placed and is followed by a glass reflector specially made to converge the light into the Fresnel lens. The converged light from the glass reflector is sent through the two Fresnel lenses and the transparent LCD. After passing through the LCD screen, the light is made to focus on the convex lens by reflecting it into the mirror. The highpower LED is powered using a power source connected to the DC-DCbuck booster. A heat sink and a cooling fan are used to reduce the heat produced by the 100W LED [4]. The experimental setup of the projector module is shown in Fig.4. 


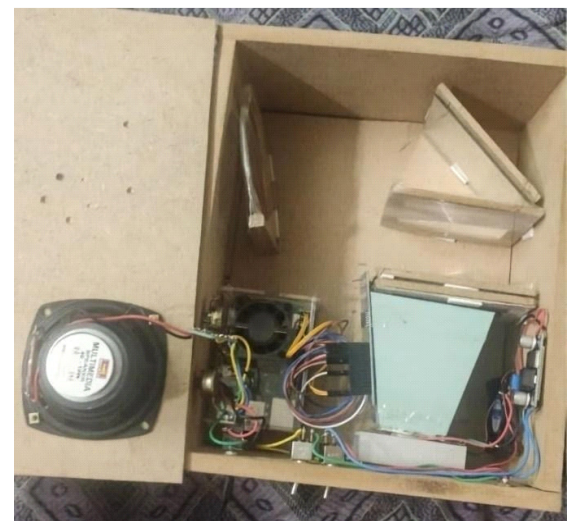

Fig.4. Experimental set up of the Projector module

\section{- Voice recognition module}

In this module, a microphone is set to a state of ever listening for commands being received from the users. The Bluetooth microphone is interfaced with Raspberry Pi and it has software consisting of Voice manipulations. It processes the voice to appropriate commands and executes them for required purposes. The lag and delays are negligible and are not considered for analysis. The important constraint is that the voice must be pronounced properly to execute the commands listed in Table 1 . The voice assistant can be terminated and started using the wake word "flora" for effective utilization and hence it is user interactive to process the commands that ever listen to the voices. This voice recognition system is developed using Python [5]. This software consists of four parts:

- Wake word engine

- Speech to text engine

- Command execution module

\section{- $\quad$ Text to Speech engine}

The wake word engine is constructed to activate the voice assistant from its sleep mode after listening to the commands from the user. According to the dictionary, a wake word is a word or phrase that activates a dormant device when spoken by the user. When the device hears the word, it begins recording, and a dark blue ring of light on the top appears. The microphones also determine the direction the word comes from and can focus in that direction. A wake-word engine is a tiny algorithm that monitors a stream of audio for a special wakeword and activates the voice assistants upon detecting it. The wake word used here is "flora" and the name of the voice assistant is named to be "bumblebee". After starting up, the voice recognition engine executes the process based upon the given commands [6].

Text To Speech (TTS) is a form of speech synthesis that converts text into spoken voice output. From the records, this system was developed initially to aid the visually impaired by offering a computer-generated spoken voice that would "read" text to the user. But it is not considered as the voice response system. Voice response systems synthesize speech by concatenating sentences from a database of pre-recorded words and are used for different purposes than TTS systems. The sentences/phrases are based on the graphemes and phonemes of a language [7]. Voice response systems are limited to synthesizing sentences that contain only words that have been predetermined by the system. TTS systems, in contrast, are 
theoretically capable of "reading" any string of text characters to form original sentences. The output of the voice recognition module can be either heard through the speaker connected to the Raspberry pi via PAM 8403 amplifier or through Bluetooth headphones. Commands given to the voice recognition module are shown in Table 1.

\section{Table 1. Command list of the voice recognition module}

\begin{tabular}{|c|c|c|}
\hline Sl. no. & COMALAND & FUNCTION \\
\hline & & MOUSE COMALANDS \\
\hline 1. & Move to $\mathrm{x} y$ & $\begin{array}{l}\text { moves the mouse to the point on the screen with } x \text {-coordinate and } y \text {-coordinate where } x \text { and } y \text { are in } \\
\text { pixels }\end{array}$ \\
\hline 2. & Move up $\mathrm{x}$ & moves the mouse up by $x$ pixels \\
\hline & Move down $\mathrm{x}$ & moves the mouse down by $\mathrm{x}$ pixels \\
\hline 4. & Move left $\mathrm{x}$ & moves the mouse left by $\mathrm{x}$ pixels \\
\hline 5. & Move right $\mathrm{x}$ & moves the mouse night by $\mathrm{x}$ pixels \\
\hline 6. & Left click & clicks the left mouse button once at the moure's curent location \\
\hline 7. & Double click & clicks the left mouve button twice at the mouve's current location \\
\hline 8. & Right click & clicks the right mouse button once at the mouse's current location \\
\hline 9. & Middle click & clicks the middle mouve button once at the mouse's current location \\
\hline 10. & Hold left & holds down the left mouse button at the mouse's current location \\
\hline 11. & Hold right & holds down the right mouse button at the mouve's curent location \\
\hline 12. & Hold middle & holds down the middle mouse button at the mouse's curent location \\
\hline 13. & Release left & releases the leff mouve button at the mouve's current location \\
\hline 14. & Release right & releases the right mouse button at the mouse's current location \\
\hline 15. & Release middle & releases the middle mouse button at the moure's curent location \\
\hline 16. & Scroll up $\mathrm{x}$ & $\begin{array}{l}\text { mouse scroll up by } \mathrm{x} \text { units. "Note that } \mathrm{x} \text { is not in pixels, and the umits scrolled will vary based on } \\
\text { operating system }\end{array}$ \\
\hline 17. & Scroll down $x$ & $\begin{array}{l}\text { mouve scroll down by } x \text { units. *Note that } x \text { is not in pixels, and the units scrolled will vary based on } \\
\text { operating system }\end{array}$ \\
\hline & & KFYBOARD COMALANDS \\
\hline 1. & Press key k1 & press and release keyboard key $(\mathrm{Kl})$ \\
\hline 2. & Hold key k1 & hold down keyboard key (K1) \\
\hline 3. & Release key k1 & release keyboard key (Kl) \\
\hline 4. & $\begin{array}{l}\text { Use shortcut } \\
\mathrm{k} 1, \mathrm{k} 2, \ldots \mathrm{k} \#\end{array}$ & press and hold keyboard keys $(\mathrm{K} 1)$ through $(\mathrm{K} \sharp)$ in order and then release them in reverse order \\
\hline 5. & Type this 'text' & type the list of words from text \\
\hline & & OTHER COMALANDS \\
\hline 1. & Word & Open the default application for word documents \\
\hline 2. & Close word & Close the currently running Word application \\
\hline 3. & Impress & Opens the default application for Power point presentations \\
\hline 4. & Close impress & Close the curently rumning Power point application \\
\hline 5. & Calculator & Opens the default calculator \\
\hline 6. & Close Calculator & \begin{tabular}{|l|} 
Closes the calculator \\
\end{tabular} \\
\hline 7. & Note & Opens the default application Notepad \\
\hline 8. & Close note & Closes the currently ruming application Notepad \\
\hline 9. & Browser & Opens the default application for browser \\
\hline 10. & Close browser & Closes the currently ruming application for browser \\
\hline 11. & Status & Gives the status of the system as a voice speech \\
\hline 12. & Battery & Gives the battery levels of the system as a voice speech \\
\hline 13. & Close & Shutdowns the entire system \\
\hline 14. & Reboot & Restarts the entire system \\
\hline 15. & Time & Gives the time in HH:MM:SS a \\
\hline 16. & Date & Gives the date in DDMMMYY as a \\
\hline 17. & Instant & Gives both date and time in standard formats as a voice speech \\
\hline 18. & Close program & Closes or terminates the voice assistant \\
\hline
\end{tabular}

\section{- Presentation module}

In this gesture-based presentation module MPU 6050, a three-axis accelerometer is used to compute the gesture values [8]. The input and output values are displayed in the OLED display. Initially, after connecting to the PC, the gesture must be recorded. So, a record option must be given and 3 gestures must be recorded (previous slide, next slide, and start presentation). After that, the Gestures must be saved in the EEPROM for future 
use. To test and use the gestures, a test gestures option should be given. The Gesture matching is done using the Dynamic Time Wrapping (DTW) algorithm [9]. The experimental setup of the gesture module is shown in Fig.5, Fig.6, and Fig.7.

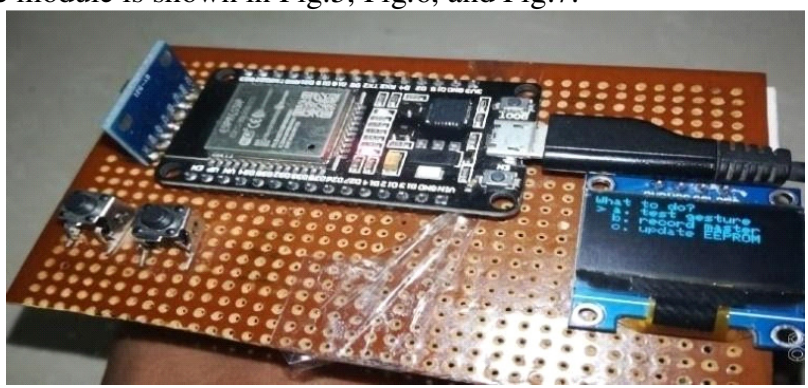

Fig.5. Implementation of Gesture module

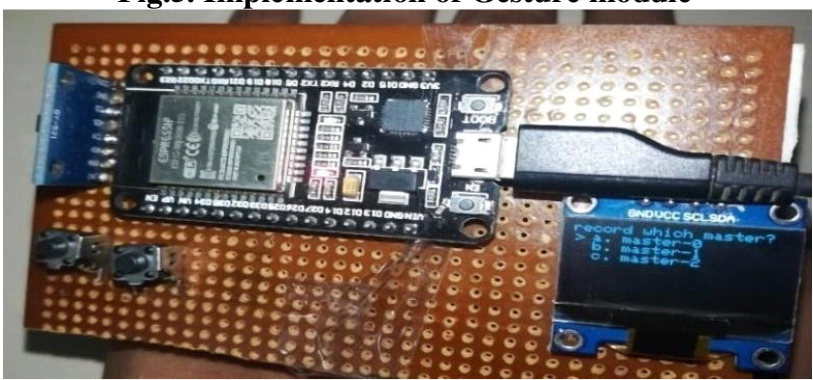

Fig.6. The gesture module is configured to record a new gesture

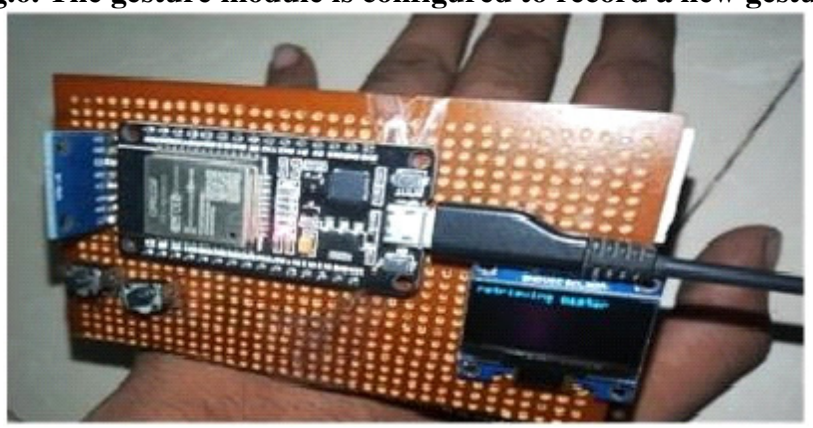

Fig.7. Saving and retrieving gesture from EEPROM - option

\section{Result Analysis}

Observations made on the projector module, voice recognition module, and gesture module are recorded. In the projector module, the high-power LED was turned ON and after turning ON the light, the image was displayed on the screen. The outputs of the projector module are shown in figures 8 and 9. 


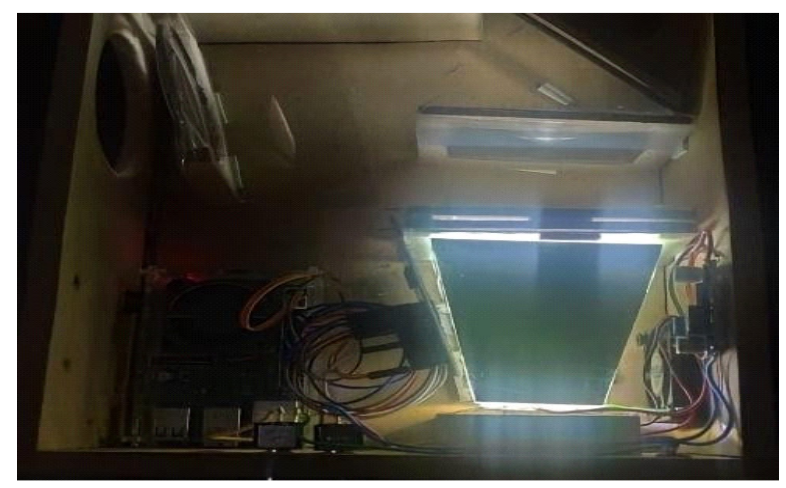

Fig.8. Status of LCD projector after turning ON the high-power LED

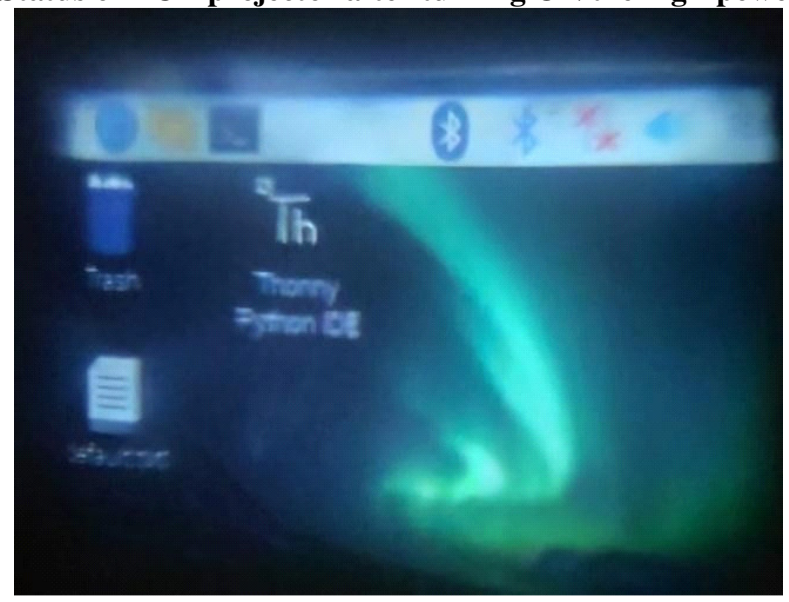

Fig.9. Projection of Desktop files on the screen

The wake-up engine gets energized on every boot of the system with the terminal running on the screen and makes the microphone wait constantly for the voice command. After saying the word "flora" the voice assistant is activated by giving a speech at the loudspeaker saying "Hello myself Bumblebee! What can I do for you?", From Table 3.1 appropriate commands can be given that turn on the specific functions. This is found to be the replacement by just voice instead of keyboards and mouse, for ease and security. The commands are given through a Bluetooth microphone connected to the Raspberry Pi and the software processes the audio and find the suitable command and execute the function. The output of the voice recognition module is shown in the below figures 10 and 11. 


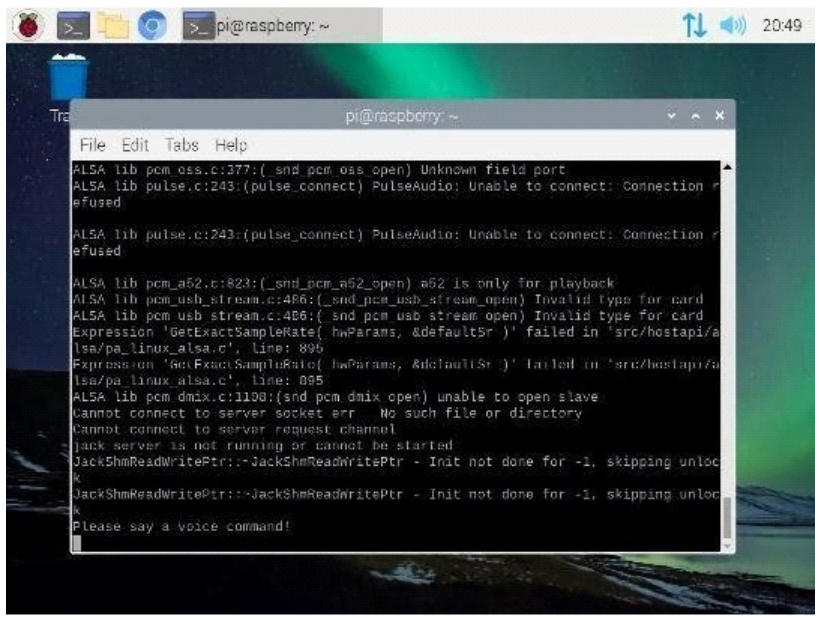

Fig.10. Activation of the wake-up engine "flora"

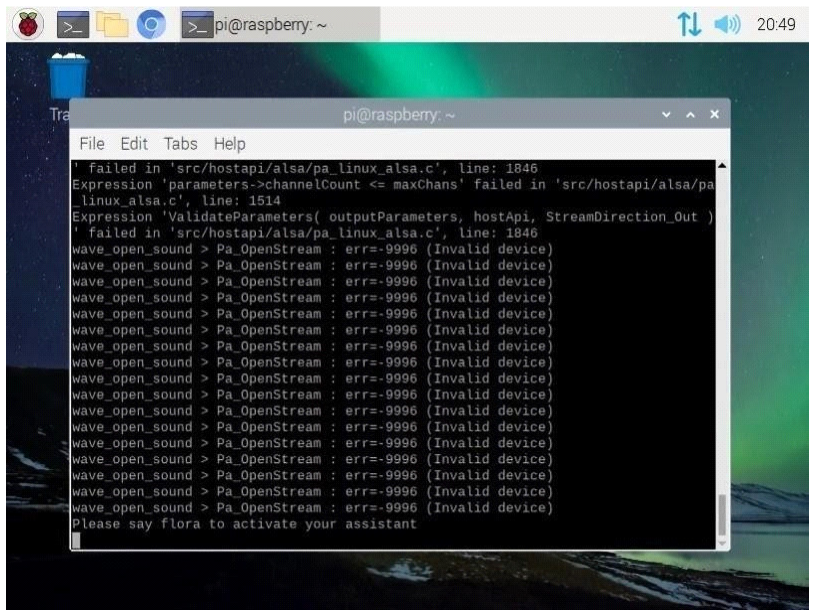

Fig.11.Activation of the voice assistant "bumblebee" 


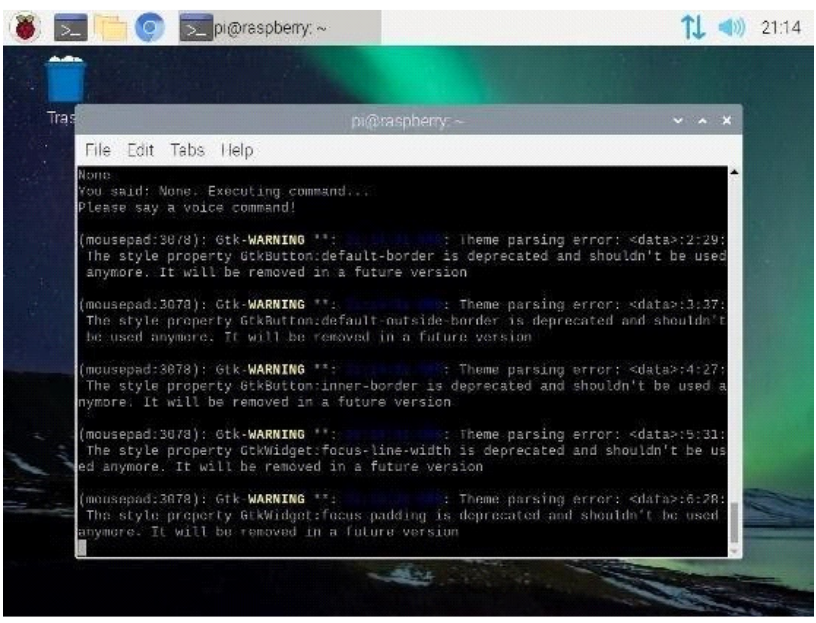

Fig.12. The command to open the notepad

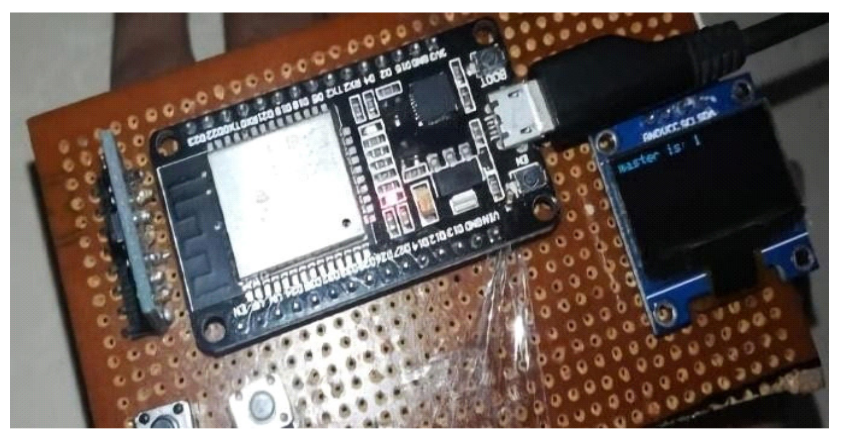

Fig.13. Voice command to type "hello world" 


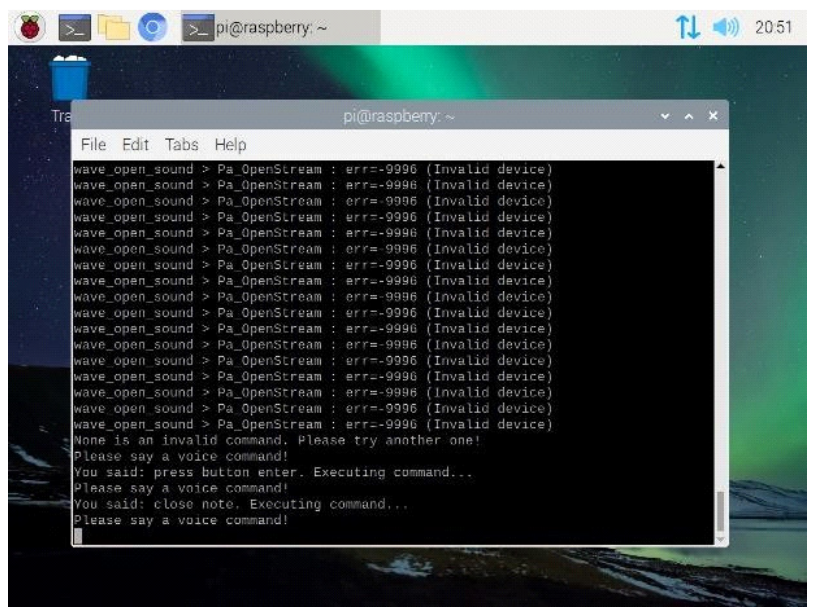

Fig.14. Voice command to close the notepad.

In the presentation module, the gestures are recorded, saved, and can retrieve from the EEPROM and are tested successfully. The output of the Gesture module is shown in figures 15,16 , and 17.

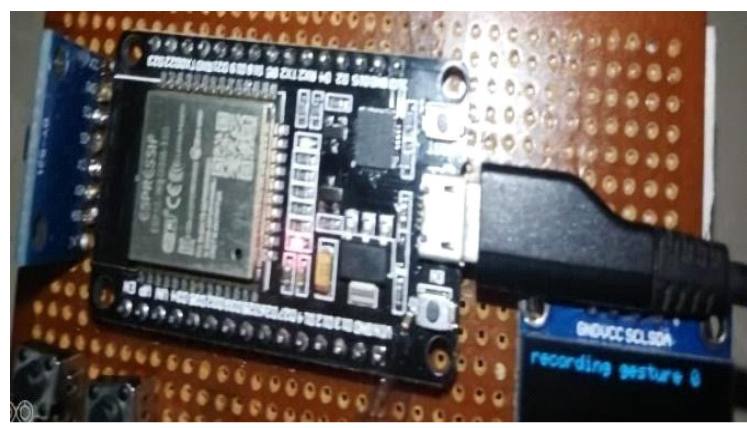

Fig.15. Recording of the gesture

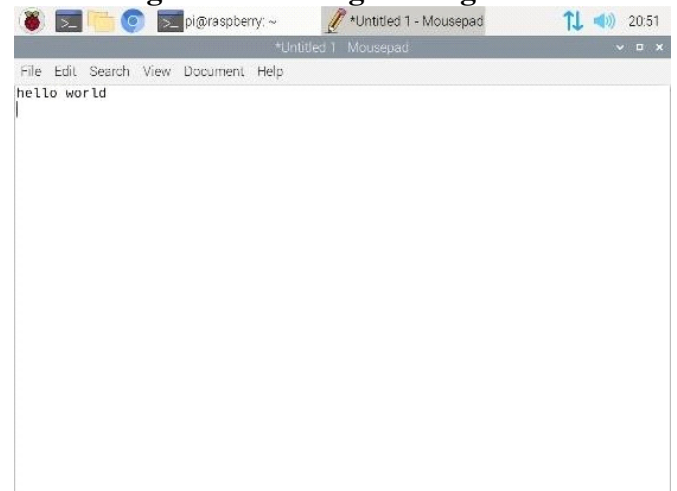

Fig.16. Successful testing of the master gesture 1 


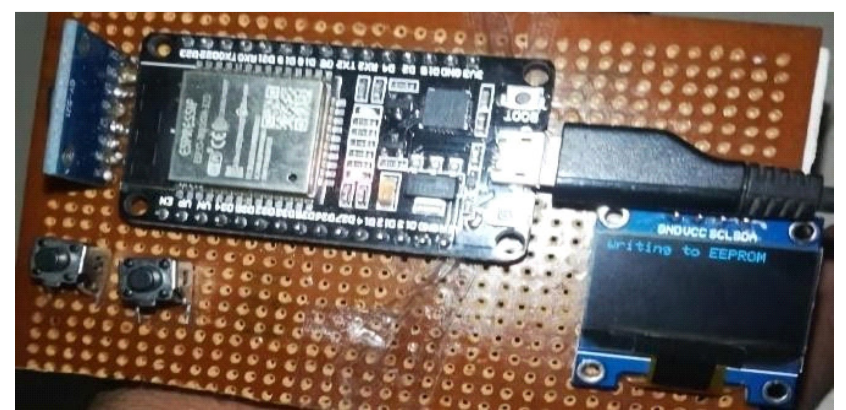

Fig.17. Saving of Master gesture in EEPROM

\section{Conclusion}

Portable and Projectable Mini PC proves to provide a solution for replacing heavy and costly projectors with low cost, lighter weight ones with the proposed novel design mechanism. The voice and hand gesture modules are integrated to introduce automation. The designed Voice recognition system is a noteworthy application for a handicapped person to use the PC with ease and the presentation module helps in interference with fewer presentations. The conventional laptops cost approximately Rs.20000. However, the proposed mini-PCs can be built at an approximate cost of Rs.8600, which reveals it as an affordable product. In the future, the prototype can be developed with miniaturized size and weight. And, the picture quality of the projector can be increased by using $4 \mathrm{~K}$ LCDs. The presentation module can be converted into an air mouse and to make the Voice recognition module access all possible applications. With these expansions, the product attains completeness.

\section{References}

[1] M. Tsuruta and N. Neubert, "An Advanced High-Resolution, High- Brightness LCD Color Video Projector," in SMPTE Journal, vol. 101, no. 6, pp. 399-403.

[2] P. Manage, V. Ambe, P. Gokhale, V. Patil, R. M. Kulkarni andP.R. Kalburgimath, "An Intelligent Text Reader based on Python," 2020 3rd International Conference on Intelligent Sustainable Systems (ICISS), 2020, pp. 1-5.

[3] H. Basanta, Y. Huang and T. Lee, "Assistive design for elderly living ambient using voice and gesture recognition system," 2017 IEEE International Conference on Systems, Man, and Cybernetics (SMC), 2017, pp. 840-845.

[4] Shabana Pirajade, Aishwarya Patil, Neha Raut, Piyush Lande, GhatikaWaykole, "LCD Projector with USB Connector", International Journal of Future Generation Communication and Networking, Vol. 13, No. 3, pp. 3252-3255.

[5] A.M.Sermakani., J.Monisha, G.Shrisha, G.Sumisha, "Creating Desktop Speech Recognization Using Python Programming", International Journal of Advanced Research in Computer and Communication Engineering, Vol. 10, Issue 3, ISSN. (Print) 2319- 5940.

[6] Mrs.P.Dhakshayeni, S. Rithika Irene, V. ThangaIshwarya, M. Vaishnavi, "Automation In Python Using Speech Recognition”, International Research Journal of Engineering and Technology, Vol. 07, Issue 3, ISSN. (Print) 2395-0072. 
[7] Vismaya V, Darvin Reynald J, "Natural Language Processing Using Python”, International Journal of Scientific \& Engineering Research, Volume 8, Issue 5, ISSN. 2229-5518.

[8] Nduanya, U. I., Oleka, C. V., Orji, E. Z., "Design and Implementation of Arduino-Based GestureControlled System with Accelerometer", International Journal of Recent Trends in Engineering and Research, Volume 04, Issue 07, ISSN. 2455- 1457.

[9] Santiago, David Pozo, Jorge Rosero, Juan Vasquez, "Gesture Recognition Using Dynamic Time Warping and Kinect: A Practical Approach", 2017 International Conference on Information Systems and Computer Science (INCISCOS).

[10] M. Tholkapiyan, A.Mohan, Vijayan.D.S , "A survey of recent studieson chlorophyll variation in Indian coastal waters”, IOP Conf. Series: Materials Science and Engineering 993 (2020) 012041 , doi:10.1088/1757-899X/993/1/012041.

[11] Lavanya Prabha, S., J. K. Dattatreya, and M. Neelamegam. "Investigation of bolted RPC plate under direct tension." Journal of Structural Engineering (Madras) 36.5 (2009): 333-341. 